\title{
Nivel de actividad física según GPAQ en mujeres embarazadas y postparto que asisten a un centro de salud familiar
}

\author{
Jaime Leppe Z., Msc ${ }^{1}$, Manuela Besomi M. ${ }^{1}$, Catalina Olsen C. $^{1}$, María Jesús Mena I. ${ }^{1}$, \\ Sonia Roa $A{ }^{1}$ \\ ${ }^{1}$ Carrera de Kinesiología, Facultad de Medicina, Clínica Alemana-Universidad del Desarrollo. Santiago, Chile.
}

\section{RESUMEN}

Antecedentes: Durante el embarazo y postparto las mujeres disminuyen el nivel de actividad física (AF). Objetivo: Determinar el nivel de AF y conducta sedentaria en mujeres embarazadas y postparto. Métodos: Participaron mujeres embarazadas y postparto entre 18 y 40 años de edad de un Centro de Salud Familiar. La AF y conducta sedentaria se midió utilizando el Global Physical Activity Questionnaire. Resultados: Se analizó la información de 47 mujeres embarazadas y 12 en postparto. La edad P50=25 años (rango:18-40 años). El $61 \%$ presentó exceso de peso. El tiempo de AF diaria fue P50=154 (rango: 0-960 min/día) en embarazadas y $\mathrm{P} 50=190$ (rango: $24-411 \mathrm{~min} / \mathrm{día}$ ) en postparto. La AF en la dimensión tiempo libre en embarazadas fue $\mathrm{P} 50=0$ (rango: 0-540 $\mathrm{min} /$ día) y $\mathrm{P} 50=3$ (rango: 0-86 min/día) en el grupo de postparto. La conducta sedentaria para todo el grupo fue P50=180 (rango: 0-720 min/día). El 20,3\% de las mujeres clasifica como suficientemente activo según criterio OMS, al corregir la AF reportada por GPAQ según IMC y nivel educacional. No hubo asociación entre las variables edad, estado nutricional, nivel educacional e ingreso familiar con el nivel de AF reportado. Conclusión: La AF de tiempo libre es una dimensión susceptible a intervenciones de promoción de la AF. El nivel de AF reportado por el grupo es mayor a resultados de estudios similares en otros países. Estudios de validación en esta población son necesarios para determinar la posible sobreestimación de los resultados por un sesgo de medición.

\section{PALABRAS CLAVE: Actividad física, salud, embarazo, postparto, GPAQ}

\section{SUMMARY}

Background: During pregnancy and postpartum, women decrease physical activity (PA) levels despite the health benefits reported in the literature for women and the fetus. Aims: To determine PA levels and sedentary behaviour in women during pregnancy and postpartum. Methods: Pregnant and postpartum women between 18 and 40 years old attending to primary care center participated. Sedentary behaviour and PA were measured using the Global Physical Activity Questionnaire (GPAQ). Results: Information was collected from 47 pregnant and 12 postpartum women. Age was $P 50=25$ (range: 18-40 years). $61 \%$ were overweight. Daily PA time was $\mathrm{P} 50=154$ (range: $0-960 \mathrm{~min} /$ day) for pregnant women and $\mathrm{P} 50=190$ (range: $24-411$ $\mathrm{min} /$ day) during postpartum. Leisure time PA was $\mathrm{P} 50=0$ (range: $0-540 \mathrm{~min} /$ day) for pregnant women and $P 50=3$ (range: 0-86 min/day) during postpartum. Sedentary behaviour was P50=180 min/day (range: 0-720 $\mathrm{min} /$ day) for all women. According to WHO criteria, only $20.3 \%$ of women are physically active, adjusting by BMI and educational level the reported PA in GPAQ. There was no association between the variables age, nutritional status, educational level, family income and reported level of PA. Conclusion: Leisure time 
PA could be susceptible to PA promotion interventions. The reported level of PA is higher than those from similar studies in other countries. Validation studies are needed to determine possible overestimation of the results due to measurement bias.

\section{KEY WORDS: Physical activity, health, pregnancy, postpartum, GPAQ}

\section{INTRODUCCIÓN}

En la última década, el tema de actividad física (AF) en relación con la mujer embarazada y en período postparto, ha sido un área más explorada. La prevalencia de mujeres insuficientemente activas de tiempo libre alcanza a un $92 \%$ a nivel país (1), esta conducta dificulta el proceso de control de ganancia excesiva de peso gestacional asociado al gasto energético a través del movimiento. Numerosos estudios han demostrado un aumento significativo de riesgos perinatales asociados a la falta de AF y al exceso de peso materno (2-5). En el primer trimestre de embarazo, la dieta vegetariana (OR=0,46; IC 95\% 0,28-0,78), el caminar (OR=0,91; IC $95 \% 0,82-1,0)$ y la $A F$ vigorosa (OR=0,76; IC $95 \% 0,6-0,97)$, tienen una asociación inversa con la ganancia excesiva de peso gestacional (3).

Según el American Congress of Obstetricians and Gynecologists, las mujeres sedentarias deben comenzar con 15 minutos de ejercicio aeróbico continuo 3 veces a la semana, aumentando a sesiones de 30 minutos 4 veces a la semana; la frecuencia cardíaca no debiera, en términos generales, superar los 140 latidos por minuto y se debe respetar las contraindicaciones relativas y absolutas propuestas por este documento (6).

La AF en la mujer embarazada ha sido estudiada, entre otros métodos, a través de cuestionarios generales como IPAQ, GPAQ y KAYSER (7-15) y cuestionarios específicos de esta población como PPAQ y PAQ (16-22). El objetivo ha sido determinar el nivel de AF y conducta sedentaria realizado por la mujer en las distintas dimensiones del movimiento humano, describiendo las variaciones presentadas durante el período de gestación. La observación mediante estos instrumentos no han estado libres de problemas de exactitud y precisión, siendo actualmente el desafío disponer de cuestionarios de auto reporte o de entrevista que posean valores de concordancia conocidos para estimar y corregir el sesgo de medición (16).

El objetivo de esta investigación es describir el nivel de AF y conducta sedentaria en las mujeres embarazadas y en período de postparto utilizando el GPAQ, el cual fue utilizado en la Encuesta Nacional de Salud 2009-2010 permitiendo comparar los resultados con patrones de referencia locales de población de mujeres sin el estado de gravidez (1). Secundariamente permitirá evaluar la aplicabilidad de este instrumento en esta población.

\section{PACIENTES Y MÉTODOS}

La investigación corresponde a un análisis secundario de datos del estudio: "Calidad de vida, problemas músculo-esqueléticos y factores asociados en la mujer embarazada y postparto", utilizando una encuesta que se llevó a cabo entre los meses de julio y septiembre del año 2011 en el Centro de Salud Familiar El Roble, comuna de La Pintana, Santiago de Chile. El estudio fue de tipo observacional, de diseño transversal. Las entrevista fueron realizadas en la zona de sala de espera del control maternal; la encuesta a utilizar fue un instrumento que recopilaba información sobre las características socio-demográficas de las mujeres, su calidad de vida, la conducta de AF, problemas músculo-esqueléticos y percepciones de salud durante el embarazo. La encuesta fue aplicada en formato "cara a cara" por un encuestador especialmente capacitado para el estudio. Toda participante debía firmar el consentimiento informado previo a la entrevista.

Para el análisis de este reporte se utilizó la información de todas aquellas mujeres embarazadas y en período postparto, entre las edades de 18 y 40 años que completaron el cuestionario GPAQ y que sus resultados fueran válidos posterior al proceso de limpieza de datos según guía de análisis de GPAQ (OMS) (23). El estudio original excluía a mujeres que estuvieran cursando embarazo múltiple, haber reportado problemas de salud o psicológicos antes o durante el embarazo, padecer una patología neurológica invalidante y cursar embarazo catalogado como de alto riesgo.

Cuestionario GPAQ: Las dimensiones de AF reportadas por el GPAQ son trabajo, desplazamiento y tiempo libre. El nivel de intensidad de la AF es clasificada en moderado o vigoroso en las dimensiones de trabajo y tiempo libre, y únicamente en nivel moderado para la dimensión desplazamiento. El cuestionario también incluye el reporte de la conducta sedentaria del participante. La determinación del nivel de AF según GPAQ se realiza según el reporte del sujeto de "un día típico de una semana típica" (23).

La clasificación de sujetos suficientemente activo se realizó según clasificación OMS de 150 minutos semanales de AF moderada, cumpliendo con bloques de a lo menos 10 minutos de AF continua (24). 
Las variables respuesta del estudio son: tiempo y nivel de intensidad de la AF total y según dimensiones, además se incluye el tiempo de conducta sedentaria. Las variables control son: edad, estado nutricional (según IMC), nivel educacional según años de estudio e ingreso familiar.

Los resultados se presentan en mediana y rango para las variables de AF debido a la condición de distribución sesgada de los datos. Se utilizó el coeficiente de correlación de Spearman para testear las variables respuestas con las variables de control. El nivel de significación fue de $5 \%$. Se utilizó el software STATA 10.0

\section{RESULTADOS}

De 105 mujeres reclutadas se analizó la información de 59 participantes, 47 embarazadas y 12 en período de postparto. La Figura 1, muestra el diagrama de flujo del proceso de reclutamiento.
La edad promedio de las mujeres fue $25 \pm 5,18$ años. El $63,6 \%$ tiene educación media completa y el $61 \%$ clasifica con exceso de peso. Todas las mujeres pertenecen al sistema FONASA. La descripción de las características socio-demográficas de la muestra según grupo se presenta en la Tabla I.

La AF diaria según GPAQ en el grupo de mujeres embarazadas fue $\mathrm{P} 50=154$ (rango: 0-960 min/ día) y P50=190 (rango: $24-411 \mathrm{~min} /$ día) en el grupo postparto. La dimensión recreacional presentó los menores valores en ambos grupos $\mathrm{P} 50=0$ y $\mathrm{P} 50=2,9$ (embarazadas y postparto, respectivamente). La dimensión trabajo reporta la mayor cantidad de minutos de AF diarios para ambos grupos. La Tabla II presenta los tiempos de AF diaria por dimensión y por grupo. Del tiempo diario total reportado el $64,4 \%$ indica realizar AF de intensidad vigorosa y un $22 \%$ de AF moderada. La distribución de las intensidades de AF según dimensión y grupos se presenta en la Tabla III.

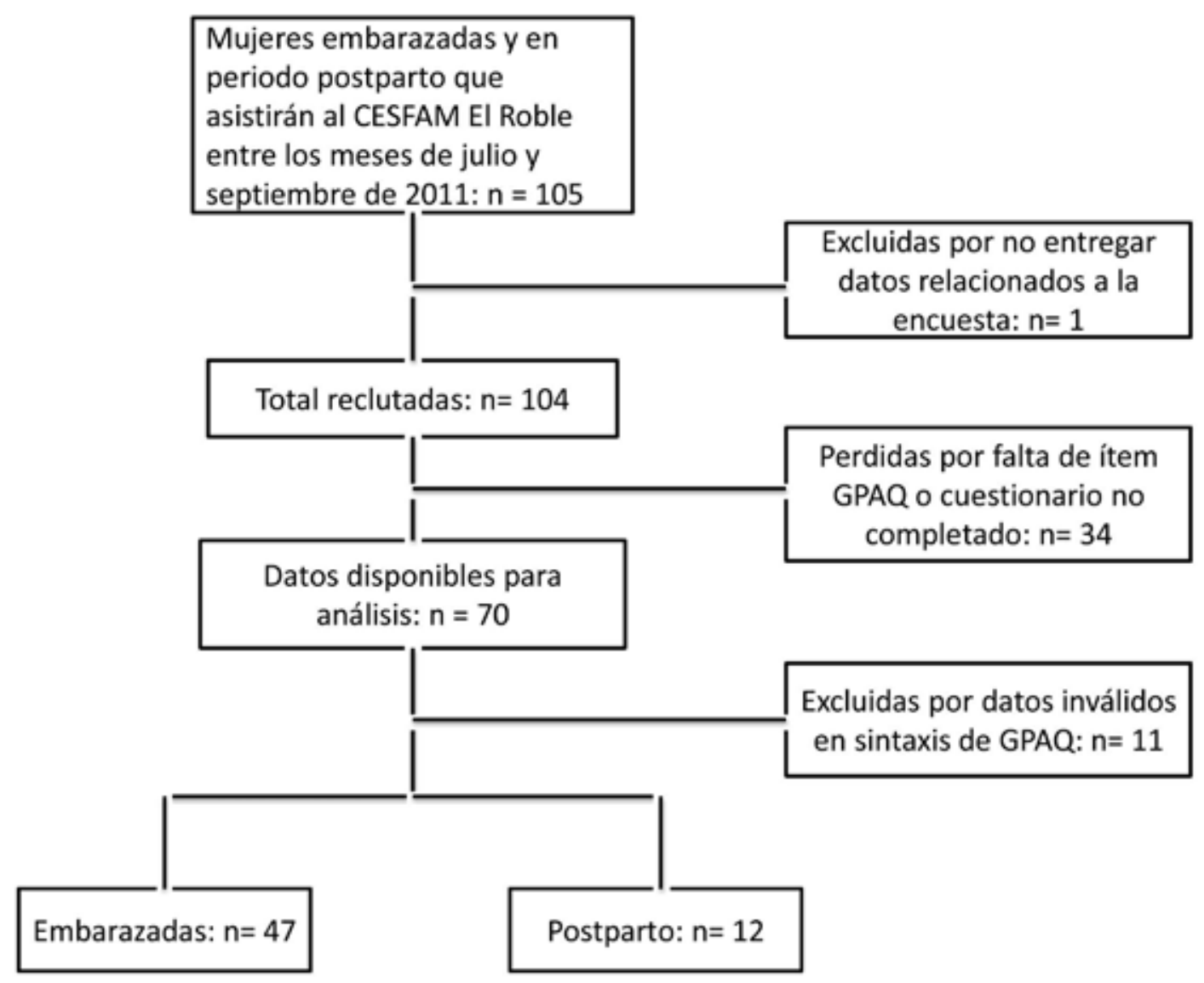

Figura 1. Diagrama de flujo del proceso de reclutamiento 
Tabla I

\section{CARACTERÍSTICAS SOCIODEMOGRÁFICAS DE MUJERES EMBARAZADAS Y EN PERÍODO POSTPARTO}

\begin{tabular}{|c|c|c|c|c|}
\hline Variables & $\mathrm{n}$ & Total $(n=59)$ & Embarazo $(e)(n=47)$ & Postparto (pp) $(n=12)$ \\
\hline $\begin{array}{l}\text { Edad * } \\
\text { Semanas e/pp * } \\
\text { Talla }(m)^{*} \\
\text { Peso (kg.) * }\end{array}$ & $\begin{array}{l}59 \\
59 \\
59 \\
59\end{array}$ & $\begin{array}{r}25(18-40) \\
22(1-39) \\
1,59(1,45-1,78) \\
71(46-140)\end{array}$ & $\begin{array}{r}25(18-40) \\
23(2-39) \\
1,59(1,45-1,78) \\
71(48-140)\end{array}$ & $\begin{array}{r}25,5(20-34) \\
14(1-36) \\
1,58(1,50-1,66) \\
67,3(46-130)\end{array}$ \\
\hline $\begin{array}{l}\text { Estado nutricional }{ }^{* *} \\
\text { Bajo peso } \\
\text { Normal } \\
\text { Sobrepeso } \\
\text { Obesidad }\end{array}$ & 59 & $\begin{array}{r}1(1,7) \\
22(37,3) \\
19(32,2) \\
17(28,8)\end{array}$ & $\begin{array}{r}0(0) \\
18(38,3) \\
15(31,9) \\
14(29,8)\end{array}$ & $\begin{array}{l}1(8,4) \\
4(33,3) \\
4(33,3) \\
3(25,0)\end{array}$ \\
\hline $\begin{array}{l}\text { Estado civil o conyugal }{ }^{\star \star} \\
\text { Casada } \\
\text { Soltera } \\
\text { Anulada } \\
\text { Otro }\end{array}$ & 59 & $\begin{array}{r}59(100) \\
12(20,3) \\
42(71,2) \\
1(1,7) \\
\quad 4(6,8)\end{array}$ & $\begin{array}{r}47(79,7) \\
10(21,3) \\
32(68,1) \\
\quad 1(2,1) \\
\quad 4(8,5)\end{array}$ & $\begin{array}{r}12(20,3) \\
2(16,7) \\
10(83,3) \\
0(0) \\
0(0)\end{array}$ \\
\hline $\mathrm{N}^{\circ}$ cohabitantes * & 59 & $4(1-10)$ & $4(1-10)$ & $5(2-8)$ \\
\hline $\mathrm{N}^{\circ}$ embarazos anteriores * & 58 & $1(0-6)$ & $1(0-6)$ & $1(0-5)$ \\
\hline $\mathrm{N}^{\circ}$ hijos vivos * & 56 & $1(0-5)$ & $1(0-3)$ & $1(1-5)$ \\
\hline Años de estudio * & 50 & $12(7-18)$ & $12(8-18)$ & $12(7-17)$ \\
\hline $\begin{array}{l}\text { Nivel educacional ** } \\
\text { Básica incompleta } \\
\text { Básica completa } \\
\text { Media incompleta } \\
\text { Media completa }\end{array}$ & 44 & $\begin{array}{r}44(100) \\
2(4,6) \\
5(11,4) \\
9(20,4) \\
28(63,6)\end{array}$ & $\begin{array}{r}33(75) \\
1(3,0) \\
5(15,2) \\
8(24,2) \\
19(57,6)\end{array}$ & $\begin{array}{r}11(25) \\
1(9,1) \\
0(0) \\
1(9,1) \\
9(81,8)\end{array}$ \\
\hline $\begin{array}{l}\text { Previsión ** } \\
\text { Fonasa } \\
\text { A } \\
\text { B } \\
\text { C } \\
\text { D }\end{array}$ & 59 & $\begin{array}{r}59(100) \\
35(59,3) \\
9(15,3) \\
10(17,0) \\
5(8,4)\end{array}$ & $\begin{array}{r}47(79,7) \\
26(55,3) \\
7(14,9) \\
10(21,3) \\
4(8,5)\end{array}$ & $\begin{array}{r}12(20,3) \\
9(75,0) \\
2(16,7) \\
0(0) \\
1(8,3)\end{array}$ \\
\hline Ingreso personal (\$ miles) * & 55 & $0(0-400)$ & $0(0-400)$ & $0(0-400)$ \\
\hline Ingreso familiar (\$ miles) * & 56 & $357,5(100-1.000)$ & $357,5(157-1.000)$ & $305(100-600)$ \\
\hline
\end{tabular}

* Datos expresados en mediana y rango P50 (rango).* Variables cualitativas expresadas en porcentaje (\%). FONASA: Fondo Nacional de Salud.

La conducta sedentaria de la población evaluada es $P 50=180$ (rango: 0-720 min/día), siendo $\mathrm{P} 50=180$ (rango: $0-120 \mathrm{~min} / \mathrm{día}$ ) y $\mathrm{P} 50=180$ (rango 0-360min/día) para el grupo de embarazadas y postparto, respectivamente.

No existe asociación entre las variables respuestas y las variables de control: edad, estado nutricional, nivel educacional e ingreso familiar $(p>0,05)$.

Según criterio OMS para clasificar como suficientemente activo aquellos sujetos que acumulan
150 minutos semanales de AF física aeróbica de intensidad moderada o 75 minutos de AF de intensidad vigorosa, o una combinación entre ambas intensidades que sean mayor a 600 METS semanales en bloques mínimo de 10 minutos continuos, el $94,9 \%$ de las participantes es considerado como suficientemente activo. Sin embargo, utilizando el modelo de corrección para la prevalencia de AF en la ENS 2009-2010 $(1,15)$ este valor corresponde a un $20,3 \%$. 
Tabla II

\section{DESCRIPCIÓN DEL TIEMPO DE ACTIVIDAD FÍSICA TOTAL Y POR DOMINIO EN EMBARAZADAS Y EN POSTPARTO (GPAQ)}

\begin{tabular}{lcc}
\hline $\begin{array}{l}\text { AF total y por dominio } \\
(\mathrm{min} / \text { día })^{\star}\end{array}$ & $\begin{array}{c}\text { Embarazadas } \\
(\mathrm{n}=47)\end{array}$ & $\begin{array}{c}\text { Postparto } \\
(\mathrm{n}=12)\end{array}$ \\
\hline AF total & $154(0-960)$ & $190(24-411)$ \\
Trabajo & $120(0-600)$ & $180(0-411)$ \\
Desplazamiento & $12,9(0-360)$ & $0(0-20)$ \\
Recreacional & $0(0-540)$ & $2,9(0-86)$ \\
\hline
\end{tabular}

*Datos expresados en mediana y rango, P50 (rango). AF: actividad física. GPAQ: Global Physical Activity Questionnaire.
Los valores de AF en la dimensión trabajo para ambos grupos son los que explican, en su gran mayoría, la clasificación de suficientemente activo según criterio OMS. Siendo el $58,6 \%$ de la población de estudio dueña de casa, es importante identificar en estudios de campo si la AF realizada en las labores de hogar puede ser clasificada como de intensidad moderada o vigorosa.

Los resultados de AF en el dominio trabajo de la ENS 2009-2010 en el subgrupo de mujeres del segmento de edad de 15-24 años reporta un promedio de $86,4 \mathrm{~min} /$ día; IC 95\% $(68,9-103,9)$ y en el segmento de 25-44 años un promedio de 141,9 $\mathrm{min} / \mathrm{día}$; IC 95\% (123,5-160,4). Los valores de nuestro estudio sólo son mayores en el segmento de post parto con un valor de referencia $P 50=180$

Tabla III

DESCRIPCIÓN DE LA INTENSIDAD DE ACTIVIDAD FÍSICA POR DOMINIO, EN EMBARAZADAS Y EN POSTPARTO (GPAQ)

\begin{tabular}{llccc}
\hline $\begin{array}{l}\text { Dominios de AF } \\
\text { (según intensidad) }\end{array}$ & $\mathrm{n}$ & Embarazadas & $\mathrm{n}$ & Postparto \\
\hline $\begin{array}{l}\text { Trabajo } \\
\quad \text { Moderado }\end{array}$ & 47 & $42(89,3)$ & 12 & $11(91,7)$ \\
$\quad$ Vigoroso & 47 & $1(2,1)$ & 11 & $0(0)$ \\
Desplazamiento moderado & 46 & $35(76,1)$ & 11 & $5(45,6)$ \\
$\begin{array}{l}\text { Actividad recreacional } \\
\quad \text { Moderado }\end{array} \quad$ & & & 12 \\
$\quad$ Vigoroso & 46 & $8(17,4)$ & 12 & $6(50,0)$ \\
\end{tabular}

*Datos expresados en porcentaje (\%).GPAQ: Global Physical Activity Questionnaire. AF: actividad física.

\section{DISCUSIÓN}

La medición de la AF en la mujer embarazada es un tema poco desarrollado en nuestra realidad nacional. El uso de instrumentos de auto reporte o entrevista son herramientas viables para el estudio de esta conducta humana. Sin embargo, el sesgo de medición de estos instrumentos ha sido frecuentemente reportado (25).

Dentro de las dimensiones de la AF el dominio "trabajo" se ha reportado con mayor grado de sobrestimación tanto en intensidad como duración en los diferentes estudios que utilizan cuestionarios como IPAQ o GPAQ. El concepto de 10 minutos continuos parece ser uno de los problemas más frecuentes que genera sobrestimación de tiempo en esta dimensión. El sujeto entrevistado generalmente sobrestima el tiempo de AF realizado o suma tiempos de intervalos menores a 10 minutos como el utilizado en el cuidado de menores. min/día, atribuidos a un valor extremo de la muestra de estudio. Es importante considerar que estos valores de referencia de la ENS 2009-2010, no incluyen mujeres embarazadas pues fue un criterio de exclusión (1).

El dominio desplazamiento muestra un resultado inferior a los $40 \mathrm{~min} /$ día reportados en la ENS 20092010 para el grupo de edad entre 15-44 años, la variable ocupación podría explicar estos resultados.

El dominio tiempo libre o recreacional $P 50=0$ $\mathrm{min} /$ día para embarazadas y p50=2,9 $\mathrm{min} /$ día en post parto, demuestra la limitada AF que esta población dedica en su tiempo libre, estos valores son consistentes con la ENS 2009-2010, donde el grupo de mujeres más activo no supera los 15 minutos en este dominio. Según la Encuesta Nacional de Hábitos de Actividad Física 2010 (25), el $52,3 \%$ de los participantes reporta no tener tiempo para practicar deporte o AF. Según este mismo instrumento en el subgrupo que dejó de realizar 
$\mathrm{AF}$, las 2 primeras razones en las mujeres son "haber dejado de asistir al colegio o liceo" $28,3 \%$ y "nacimiento de una hijo/a" 18,6\%. El factor colegio en el segmento socioeconómico D corresponde a un $39,9 \%(25)$.

La conducta sedentaria del grupo parece ser un resultado bajo, 3 horas al día de conducta sedente implican necesariamente no tener una actividad laboral que implique actividades laborales en posición sedente. Esto podría explicarse por la mayor cantidad de actividades relacionadas con las actividades de dueña de casa. Es importante destacar que el cuestionario GPAQ reporta la dimensión trabajo para actividades como estudiar, cuidado del hogar o trabajo remunerado.

El GPAQ está siendo utilizado muy recientemente en poblaciones especiales como las embarazadas. Tendais y cols (26), midieron la AF, calidad de vida relacionada a la salud y depresión desde el pre-embarazo hasta el segundo trimestre gestacional utilizando GPAQ en una muestra de 56 participantes. Sus resultados muestran una disminución en la cantidad total de AF $(p<0,002)$ e intensidad vigorosa $(p<0,011)$ de la dimensión tiempo libre entre el pre-embarazo y los dos primeros trimestres. No encontraron un cambio en el dominio trabajo ni desplazamiento. Los resultados de Tendais y cols (26), son menores a los reportados en este estudio, probablemente debido a las diferencias en las características socio-demográficas de sus participantes, especialmente el mayor nivel educacional.

Otros instrumentos, como el IPAQ, han sido utilizados para medir la AF en embarazadas. Según este mismo instrumento en el estudio de Harrison y cols (27), la AF reportada en METs y no en minutos semana, reporta valores promedio de 285,26 METS.min-1, en el caso de reportar nuestros resultados en METs, estos corresponden al 50\% más que los de Harrison y cols. Esta diferencia podría explicarse por la posible sobrestimación mencionada anteriormente en la dimensión trabajo.

Es importante considerar que las características socio-demográficas de nuestra población de estudio, principalmente en su variable nivel educacional e IMC la hacen susceptible de presentar un sesgo de medición sobrestimando los resultados de su AF realizada en sus actividades cotidianas. La evaluación de la validez y confiabilidad de la aplicación del GPAQ en la ENS 2009-2010 demostró que la sobrestimación del cuestionario comparado con la medición objetiva efectuada con acelerómetros presentó un sobre reporte promedio de 548 minutos por semana, esta sobrestimación estaba asociada con ser: sexo hombre, tener menos de 8 años de educación y mayor IMC (15). Por lo tanto, al aplicar el modelo de corrección utilizado en la ENS 2009$2010(1,15)$ el valor de suficientemente activo se reduce a sólo un $20,3 \%$.
La importancia de realizar estudios de validación con mediciones objetivas para evaluar la concordancia entre los instrumentos es una necesidad para determinación del sesgo de medición y la adecuada interpretación de los resultados en la aplicación de políticas de salud en este grupo. El estudio "Asociación entre actividad física y salud en la mujer embarazada: una línea de base para futuras intervenciones", proyecto FONIS SA11I2161 en ejecución, tiene entre otros objetivos evaluar la validez de los cuestionarios GPAQ y PPAQ en una población de similares características socio-demográficas a las de este estudio, lo que podría informar sobre el tamaño del sesgo de reporte.

El nivel de AF reportado por los grupos es mayor a los resultados de estudios similares en el extranjero, probablemente por diferencias culturales y socio-demográficas especialmente el nivel educacional con nuestra población estudiada. El mayor reporte de AF en cuanto a tiempo en minutos se concentró en la dimensión trabajo. A pesar de que estos datos son consistentes con la ENS 2009-2010, es importante considerar que las mujeres podrían sobrestimar dicha actividad, sobre todo aquellas que poseen un bajo nivel educacional y mayor IMC.

La actividad de tiempo libre o recreacional resulta ser una dimensión susceptible a intervenciones de promoción de la AF. Los determinantes sociales de la población en estudio: ser mujer, bajo ingreso personal y familiar, edad de maternidad cercana al período de egreso del colegio o liceo, exceso de peso y su nivel educacional, los disponen a ser un grupo altamente probable de no realizar AF o abandonar este hábito si antes del embarazo lo poseía.

\section{CONCLUSIÓN}

La utilización de cuestionarios de vigilancia epidemiológica o de uso clínico para la medición de la AF en embarazadas debe ser estudiado a través de mediciones objetivas que reporten el sesgo de medición de las entrevistadas para permitir obtener mediciones más exactas y precisas para utilizar en la decisión de la atención clínica y promoción de estilos de vida saludable en esta población.

\section{REFERENCIAS}

1. MINSAL. Objetivos estratégicos en salud. Revisión Final. Hallado en:www.redsalud.gov.cl/portal/url/item/94 a33f151ef4a574e04001011f0131dd.pdf. Acceso el 29 deMayo 2010.

2. Márquez J, García V, Ardila R. Ejercicio y prevención de obesidad y diabetes mellitus gestacional. Rev Chil Obstet Ginecol 2012;77(5):401-6.

3. Stuebe A, Oken E, Gillman M. Associations of diet and physical activity during pregnancy with risk for excessive gestational weight gain. AmJ Obstet Gynecol 2009;201(1):58.e1-8. 
4. De la Calle M, Armijo M, Martín E, Sancha M, Magdaleno $\mathrm{F}$, Omeñaca $\mathrm{F}$, et al. Sobrepeso y obesidad pregestacional como factor de riesgo de cesárea y complicaciones perinatales. Rev Chil Obstet Ginecol 2009;74(4):233-8.

5. Atalah E, Castro R. Obesidad materna y riesgo reproductivo. Rev Med Chile 2004;132:923-30.

6. Davies GA, Wolfe LA, Mottola MF, MacKinnon C. Exercise in pregnancy and the postpartum period. Can J Appl Physiol 2003;28(3):330-41.

7. Bull FC, Maslin TS, Armstrong T. Global Physical Activity Questionnaire (GPAQ): Nine country realiability and validity study. J Phys Act Health 2009;6(6):790804.

8. Armstrong T, Bull FC. Development of the World Health Organization Global Physical Activity Questionnaire (GPAQ). J Public Health 2006;14(2):66-70.

9. Au TB, Blizzard L, Schmidt M, Pham LH, Magnussen $\mathrm{C}$, Dwyer T. Reliability and validity of the global physical activity questionnaire in Vietnam. J Phys Act Health 2010;7(3):410-8.

10. Hoos T, Espinoza N, Marshall S, Arredondo EM. Validity of the Global Physical Activity Questionnaire (GPAQ) in Adult Latinas. J Phys Act Health 2012;9(5):698-705.

11. Trinh OT, Nguyen ND, van der Ploeg HP, Dibley MJ, Bauman A. Test-Retest repeatability and relative validity of the Global Physical Activity Questionnaire in a developing country context. J Phys Act Health 2009;6 Suppl 1:S46-53.

12. Craig CL, Marshall AL, Sjöström M, Bauman AE, Booth $\mathrm{ML}$, Ainsworth BE, et al. International physical activity questionnaire: 12-country reliability and validity. Med Sci Sports Exerc 2003;35(8):1381-95.

13. Ng N, Hakimi M, Van Minh H, Juvekar S, Razzaque A, Ashraf $A$, et al. Prevalence of physical inactivity in nine rural INDEPTH Health and Demographic Surveillance Systems in five Asian countries. Glob Health Action 2009;28(2) Suppl 1:44-53.

14. Leppe J, Margozzini P, Villarroel L, Sarmiento OL, Guthold R, Bull FC. Validity of the global physical activity questionnaire in the National Health Survey-Chile 2009-10. J Sci Med Sport 2012;15:Suppl 1:S297.

15. Chasan-Taber L, Silveira M, Marcus BH, Braun B, Stanek E, Markenson G. Feasibility and efficacy of a physical activity intervention among pregnant women: the behaviors affecting baby and you (B.A.B.Y.) study. J Phys Act Health 2011;8 Suppl 2:S228-38.

16. Foxcroft KF, Rowlands IJ, Byrne NM, Mclntyre HD, Callaway LK. Exercise in obese pregnant women: the role of social factors, lifestyle and pregnancy symptoms. BMC Pregnancy Childbirth 2011.Disponible en: www.biomedcentral.com/1471-2393/11/4/.Acceso el 17 de Enero 2011.

17. Söhnchen N, Melzer K, Tejada BM, Jastrow-Meyer N, Othenin-Girard V, Irion $\mathrm{O}$. Maternal heart rate changes during labour. Eur J Obstet Gynecol Reprod Biol 2011;158(2):173-8.

18. Haakstad LA, Gundersen I, Bø K. Self-reporting compared to motion monitor in the measurement of physical activity during pregnancy. Acta Obstet Gynecol Scand 2010;89(6):749-56.

19. McParlin C, Robson SC, Tennant PW, Besson H, Rankin J, Adamson AJ, et al. Objectively measured physical activity during pregnancy: a study in obese and overweight women. BMC Pregnancy Childbirth 2010. Disponible en: www.biomedcentral.com/14712393/10/76/prepub. Acceso el 30 de Noviembre 2010.

20. Matsuzaki M, Haruna M, Ota E, Yeo S, Murayama R, Murashima S. Translation and cross-cultural adaptation of the Pregnancy Physical Activity Questionnaire (PPAQ) to Japanese. Biosci Trends 2010;4(4):170-7.

21. Evenson KR. Towards an Understanding of Change in physical activity from pregnancy through postpartum. Psychol Sport Exerc 2011;12(1):36-45.

22. Global Physical Activity Questionnaire. Department of Chronic Diseases and Health Promotion. Hallado en: www.who.int/chp/steps/GPAQ/en/index.html. Acceso el 18 de Julio de 2012.

23. Organización Mundial de la Salud (OMS). Recomendaciones mundiales sobre la Actividad Física para la salud, 2010. Hallado en: www.who.int/dietphysicalactivity/factsheet_recommendations/es/. Acceso el 18 de Julio de 2012.

24. Encuesta Nacional de Hábitos de Actividad Física (ENHAF), 2010. Hallado en:http://www.ind.cl/Documents/ Seminario\%20Chile\%2010/Hacia-Politica-Deportiva/ Encuesta\%20Nacional\%20de\%20Habitos\%202009. pdf. Acceso el 16 de Julio de 2012

25. Hallal PC, Gómez LF, Parra DC, Lobelo F, Mosquera $\mathrm{J}$, Florindo $\mathrm{AA}$, et al. Lessons learned after 10 years of IPAQ use in Brazil and Colombia. J Phys Act Health 2010;7 Suppl 2:S259-64.

26. Tendais I, Figueiredo B, Mota J, Conde A. Physical activity, health-related quality of life and depression during pregnancy. Cad Saude Publica 2011;27(2):219-28.

27. Harrison CL, Thompson RG, Teede HJ, Lombard CB. Measuring physical activity during pregnancy. Int $\mathrm{J}$ Behav Nutr Phys Act 2011;8:19. Disponible en: http:// www.ijbnpa.org/content/8/1/19. Acceso el 22 de Marzo de 2011. 